\title{
A global asymptotic stability condition for a dimorphic Lotka-Volterra model with explicit indirect interactions
}

\author{
Mircea T. Sofonea ${ }^{1,2}, *$ \\ 1MIVEGEC, CNRS, IRD, Université de Montpellier - 911 avenue Agropolis, B.P . 64501, 34394 \\ Montpellier Cedex 5, France \\ 2 Université de Montpellier, place Eugène Bataillon, Montpellier, France \\ * Corresponding author (mircea.sofonea@normalesup.org)
}

\begin{abstract}
A two-species Lotka-Volterra model extended with an arbitrary number of indirect interactions through diffusible and renewable compounds is presented according to its relevance in microbial community modelling. After the determination of the system's fixed points and a short discussion over their local asymptotic stability, Lyapunov's second method is applied to derive a sufficient condition of global asymptotic stability. Biologically, this condition indicates the necessity for one microbial type to show strong self-inhibition and the compounds to be fastly replaced.
\end{abstract}

10

Keywords: global stability, Lyapunov's Second Method, competitive Lotka-Volterra, exploitative competition, public goods, spite. 


\section{Introduction}

investigated by determining their fixed points and studying their stability after linearisation in their neighbourhood $[1,2]$. This straightforward method, however, cannot be applied to non hyperbolic fixed points and is usually inefficient to investigate the dynamics far from and between fixed points. Linearisation is especially unable to predict which locally asymptotically stable (LAS) fixed point the solution will eventually converge to or to detect non fixed point attractors such as limit cycles or chaos. These questions can be addressed using a global stability analysis, for which a suitable approach is known as Lyapunov's Second Method [3]. This method is based on the definiteness properties of a so-called Lyapunov function that needs to be identified for each fixed point of the dynamical system under study. The existence of such a function thus provides a sufficient condition for the global stability of the system.

Lyapunov functions have been widely used in mathematical biology and especially to model ecological communities $[4,5]$ for two reasons. The first one is that the dynamics of these systems emerge from the interactions between numerous individuals hence, assuming a well-mixed community, the encounters follow the classical non-linear mass action law $[6,7]$. The second reason is that biologists seek for global asymptotic stability because it guarantees the convergence of the systems towards the same point whatever the initial conditions (which are poorly known in natural phenomena). Moreover, if a closed-form value of this globally asymptotically stable (GAS) fixed point can be found, the focal dynamics can be nested into long-term processes such as evolution, by means of a timescale separation assumption $[8,9]$.

Since their first formulations $[10,11]$, the Lotka-Volterra equations have been a fruitful source of models and generalizations and constitute undoubtedly one of the most studied theoretical frameworks in population biology and even beyond [5, 12]. Nowadays, Lotka-Volterra equations continue to receive a strong interest in the study of the stability of ecological communities [13] and are especially investigated within the popular topic of microbiome $[14,15]$.

In contrast with macroscopic free living organisms that often interact directly by contact (predation, competition or mutualism) [16], an important part of the interactions between symbiotic micro-organisms, may they be commensal, mutualist or parasitic, is carried through soluble compounds secreted in the medium which may have negative as well as positive effects on their growth $[17,18,19]$.

Some bacteria for instance, secrete siderophores chelating molecules that harvest iron in the medium, which can be then imported within the cell provided that the bacterium possesses the 
matching receptor $[20,21]$. Cheating bacteria may not produce siderophores but still benefit from the siderophores produced by other bacteria [22]. On the other side, bacteriocins are secreted enzymes that break the bacterial wall. This detrimental compound may even affect the producing bacteria [23]. If pathogenic, the reproduction of the bacteria elicits the production of host defense molecules such as lactoferrin and siderocalin [24]. Analogous examples can be found for other microbes such as virus and protozoans [17]. It results from here that the dynamics of microbial communities are not shaped by simple direct competitive interactions but by a great set of indirect interactions with various effects on each microbe type $[25,26]$. Such indirect interactions are lacking in the Lotka-Volterra equations, which would need structural modifications to fit to microbial dynamics.

In this article, we consider the classical Lotka-Volterra equations to which we add an arbitrary number of indirect interactions through diffusible compounds, for which we seek a sufficient global stability condition and biologically interpret its closed-form expression. We restrict the model to two microbe types in order to derive the less stringent condition suited for major theoretical ecology and evolutionary biology frameworks that rely - by virtue of their game theory origins [?, 8]. 


\section{Model formulation}

Let us consider two microbe types (may they be strains or species) labeled 1 and 2. Hereafter, unless stated otherwise, $i$ will indifferently refer to 1 or 2 and $k$ to the other type - that is the quantification $\forall(i, k) \in\{1,2\}^{2}, i \neq k$ will be implied or simply reminded as $i=1,2 ; k=2,1$.

The microbial densities are non-negative variables $X_{i} \in \mathbb{R}_{+}$that vary through time $t \in \mathbb{R}_{+}$ according to several processes. The first is an intrinsic growth quantified by the rate $\varrho_{i} \in \mathbb{R}$, which can be negative if the microbe is unable to grow without collective help. The second is the self density dependent effect emerging from direct interactions between individuals that belong to the same type and is quantified by $\eta_{i, i} \in \mathbb{R}$. Likewise, a cross density dependent effect can be defined as describing the consequences of the inter-type direct interactions and is quantified by $\eta_{i, k} \in \mathbb{R}$ where the first index indicates the type that undergoes the interaction and the second index the one that produces it.

Finally, we consider that an arbitrary number $n \in \mathbb{N}$ of diffusible compounds modulate microbial growth according to their non-negative concentration $Y_{p} \in \mathbb{R}_{+}$and their respective effect $\gamma_{i, p} \in \mathbb{R}$ on microbial type $i$ 's growth. Whether they are microbial or host secretions, these compounds are likely to be produced at non-negative rates that are proportional to microbial densities through a positive factor denoted by $u_{i, p} \in \mathbb{R}_{+}$. Note that $u_{i, p}=0$ if microbial type $i$ does not contribute to compound $p$ production. We moreover assume that these compounds are mostly removed from the medium through processes that are not related to their activity, such as self-denaturation, host degradation, dilution which is the case of a large class of secreted molecules namely those with a renewable activity such as enzymes (bacteriocins) or chelates (siderophores, lactoferrin). Their clearance is therefore proportional to their concentration through a positive factor denoted by $v_{p}>0$ [27]. In the end, the microbial growth of the two types conforms to the following set of ODEs, with $i=1,2 ; k=2,1 ; p=1, \ldots, n$ :

$$
\begin{cases}\frac{\mathrm{d} X_{i}}{\mathrm{~d} t}=\left(\varrho_{i}+\eta_{i, i} X_{i}+\eta_{i, k} X_{k}+\sum_{p=1}^{n} \gamma_{i, p} Y_{p}\right) X_{i} & =: f_{i}(\mathbf{w}), \\ \frac{\mathrm{d} Y_{p}}{\mathrm{~d} t}=u_{i, p} X_{i}+u_{k, p} X_{k}-v_{p} Y_{p}, & =: g_{p}(\mathbf{w}),\end{cases}
$$

where $\mathbf{w}:=\left[\begin{array}{lllll}X_{1} & X_{2} & Y_{1} & \cdots & Y_{n}\end{array}\right]^{\mathrm{T}}$. For the sake of concision, we hereafter denote by $R_{i}(\mathbf{w})$ the quotient $f_{i}(\mathbf{w}) / X_{i}$ which can be interpreted as the instantaneous per capita growth rate of microbial 
type $i$. The Jacobian matrix associated to (1) is

$$
\begin{aligned}
\mathbf{J}(\mathbf{w}) & :=\frac{\partial}{\partial \mathbf{w}}\left[\begin{array}{lllll}
f_{1} & f_{2} & g_{1} & \cdots & g_{n}
\end{array}\right](\mathbf{w}), \\
= & {\left[\begin{array}{ccccc}
R_{1}(\mathbf{w})+\eta_{1,1} X_{1} & \eta_{1,2} X_{1} & \gamma_{1,1} X_{1} & \cdots & \gamma_{1, n} X_{1} \\
\eta_{2,1} X_{2} & R_{2}(\mathbf{w})+\eta_{2,2} X_{2} & \gamma_{2,1} X_{2} & \cdots & \gamma_{2, n} X_{2} \\
u_{1,1} & u_{2,1} & -v_{1} & \mathbf{0}_{p-2}^{\mathrm{T}} & 0 \\
\vdots & \vdots & \mathbf{0}_{p-2} & \ddots & \mathbf{0}_{p-2} \\
u_{1, n} & u_{2, n} & 0 & \mathbf{0}_{p-2}^{\mathrm{T}} & -v_{p}
\end{array}\right], }
\end{aligned}
$$

where $\mathbf{0}_{d}$ denotes the zero vector of $\mathbb{R}^{d}$.

Because of the biological meaning of variables $X_{i}$ and $Y_{i}$, we restrict the initial conditions (considered at $t=0)$ of (1) to the positive orthant $\mathcal{W} \equiv\left(\mathbb{R}_{+}\right)^{p+2}$. It is straightforward to see that $\mathcal{W}$ is positively invariant. Indeed, $X_{i}(t)=X_{i}(0) \exp \left(\int_{0}^{t} R_{i} \mathrm{~d} \tau\right)$, therefore $X_{i}(t) \geq 0, \forall t \geq 0$. Therefore, $\frac{\mathrm{d} Y_{p}}{\mathrm{~d} t} \geq-v_{p} Y_{p}$, from which it follows that $Y_{p}(t) \geq Y_{p}(0) \exp \left(-v_{p} t\right) \geq 0, \forall t \geq 0$. Hereafter, global properties are understood with respect to $\mathcal{W}$. 


\section{Fixed point determination}

The sets $\mathcal{X}_{i}:=\left\{\mathbf{w} \in \mathbb{R}^{p+2}: f_{i}(\mathbf{w})=0\right\}$ and $\mathcal{Y}_{p}:=\left\{\mathbf{w} \in \mathbb{R}^{p+2}: g_{p}(\mathbf{w})=0\right\}$ define the $i$-th microbial density nullcline and the $p$-th compound concentration nullcline respectively. A fixed point $\mathbf{w}$ of (1) is such that $\forall(i, p) \in\{1,2\} \times\{1, \ldots, n\}, f_{i}(\mathbf{w})=0=g_{p}(\mathbf{w})$. The set of fixed points $\mathcal{F}$ is thus the intersection of all nullclines [28], $\mathcal{F}=\mathcal{X}_{1} \cap \mathcal{X}_{2} \cap \bigcap_{p=1}^{n} \mathcal{Y}_{p}$.

On one hand, all points of $\mathcal{F}$ satisfy

$$
Y_{p}=\frac{1}{v_{p}}\left(u_{1, p} X_{1}+u_{2, p} X_{2}\right), \quad p=1, \ldots, n
$$

which allows us to rewrite $R_{i}$ on $\mathcal{F}$ such as

$$
\mathbf{w} \in \mathcal{F} \Longrightarrow R_{i}(\mathbf{w})=\varrho_{i}+m_{i, i} X_{i}+m_{i, k} X_{k}, \quad i=1,2, k=2,1,
$$

with

$$
m_{i, j}:=\eta_{i, j}+\sum_{p=1}^{n} \frac{\gamma_{i, p} u_{j, p}}{v_{p}}
$$

On the other hand, all points of $\mathcal{F}$ satisfy $\left(X_{i}=0\right) \vee\left(R_{i}=0\right)$ for $i=1,2$, where $\vee$ denotes the logical disjunction (or). By canceling either $X_{i}$ or $R_{i}$ for each type, it follows that there exist at most

$$
\begin{aligned}
& \mathbf{w}_{2}=\left[\begin{array}{lllll}
0 & x_{2} & \frac{u_{2,1} x_{2}}{v_{2}} & \cdots & \frac{u_{2, n} x_{2}}{v_{n}}
\end{array}\right]^{\mathrm{T}} \text {, where } x_{2}:=-\frac{\varrho_{2}}{m_{2,2}} \text {, provided th } \\
& \text { The fourth fixed point is the dimorphic fixed point } \mathbf{w}_{3} \text {, which satisfies } \\
& \text { equations represent the linear system } \\
& \qquad\left[\begin{array}{ll}
m_{1,1} & m_{1,2} \\
m_{2,1} & m_{2,2}
\end{array}\right] \cdot\left[\begin{array}{l}
X_{1} \\
X_{2}
\end{array}\right]=-\left[\begin{array}{l}
\varrho_{1} \\
\varrho_{2}
\end{array}\right],
\end{aligned}
$$
by solving the system $\left(R_{1}=0\right) \wedge\left(X_{2}=0\right)$, where $\wedge$ denotes the logical conjunction (and). Hence, $\mathbf{w}_{1}=\left[\begin{array}{lllll}x_{1} & 0 & \frac{u_{1,1} x_{1}}{v_{1}} & \cdots & \frac{u_{1, n} x_{1}}{v_{n}}\end{array}\right]^{\mathrm{T}}$, where $x_{1}:=-\frac{\varrho_{1}}{m_{1,1}}$ is the stationary $i$-th monomorphic microbial density, provided that $m_{1,1} \neq 0$ (hereafter assumed). Analogously, the monomorphic fixed point 2 is $\mathbf{w}_{2}=\left[\begin{array}{lllll}0 & x_{2} & \frac{u_{2,1} x_{2}}{v_{2}} & \cdots & \frac{u_{2, n} x_{2}}{v_{n}}\end{array}\right]^{\mathrm{T}}$, where $x_{2}:=-\frac{\varrho_{2}}{m_{2,2}}$, provided that $m_{2,2} \neq 0$ (hereafter assumed). The fourth fixed point is the dimorphic fixed point $\mathbf{w}_{3}$, which satisfies $\left(R_{1}=0\right) \wedge\left(R_{2}=0\right)$. These two

the solutions of which is $\left[\begin{array}{ll}\xi_{1} & \xi_{2}\end{array}\right]^{\mathrm{T}}$ with $\xi_{i}:=\frac{m_{i, k} \varrho_{k}-m_{k, k} \varrho_{i}}{m_{1,1} m_{2,2}-m_{1,2} m_{2,1}}$, provided that $m_{1,1} m_{2,2} \neq m_{1,2} m_{2,1}$ 115 (hereafter assumed). It results from above that $\mathbf{w}_{3}=\left[\begin{array}{lllll}\xi_{1} & \xi_{2} & \frac{1}{v_{1}}\left(u_{1,1} \xi_{1}+u_{2,1} \xi_{2}\right) & \cdots & \frac{1}{v_{n}}\left(u_{1, n} \xi_{1}+u_{2, n} \xi_{2}\right)\end{array}\right]^{\mathrm{T}}$.

We say that a fixed point is feasible when it belongs to $\mathcal{W}$. While $\mathbf{w}_{0}$ is always feasible, the three 
other fixed points may not. Their feasibility conditions are $\operatorname{sgn} \varrho_{1} \neq \operatorname{sgn} m_{1,1}, \operatorname{sgn} \varrho_{2} \neq \operatorname{sgn} m_{2,2}$ and $\operatorname{sgn}\left(m_{1,2} \varrho_{2}-m_{2,2} \varrho_{1}\right)=\operatorname{sgn}\left(m_{2,1} \varrho_{1}-m_{1,1} \varrho_{2}\right)=\operatorname{sgn}\left(m_{1,1} m_{2,2}-m_{1,2} m_{2,1}\right)$ respectively, where sgn is the sign function. 


\section{Local asymptotic stability}

Fixed point local asymptotic stability is addressed, as classically, through the sign of the real parts of the eigenvalues of the Jacobian matrix evaluated at the given point. In the case of the null fixed point, $\mathbf{J}\left(\mathbf{w}_{0}\right)$ is a lower triangular matrix the diagonal of which is simply $\left[\begin{array}{lllll}\varrho_{1} & \varrho_{2} & -v_{1} & \cdots & -v_{p}\end{array}\right]^{\mathrm{T}}$ hence $\mathbf{w}_{0}$ is LAS whenever $\left(\varrho_{1}<0\right) \wedge\left(\varrho_{2}<0\right)$.

One can also derive the necessary and sufficient condition of local asymptotic stability of the monomorphic fixed points $\mathbf{w}_{1}$ and $\mathbf{w}_{2}$ provided that they are LAS in their respective monomophic space $\mathcal{W}_{i}:=\left(\mathcal{W} \cap \mathcal{X}_{k}\right) \backslash \mathcal{X}_{i}$. (Note that $\mathcal{W}_{i}$ is the subspace of $\mathcal{W}$ where the focal microbial density is positive while the other microbial type is constrained to zero.) Indeed, $\mathbf{J}\left(\mathbf{w}_{2}\right)$ is a lower triangular block matrix of the form

$$
\left[\begin{array}{cc}
R_{1}\left(\mathbf{w}_{2}\right) & \mathbf{0}_{p+1} \\
\mathbf{u}_{1} & \mathbf{J}_{1,1}\left(\mathbf{w}_{2}\right)
\end{array}\right]
$$

where $\mathbf{u}_{1}=\left[\begin{array}{lll}u_{1,1} & \cdots & u_{1, p}\end{array}\right]^{\mathrm{T}}$ and $\mathbf{J}_{1,1}\left(\mathbf{w}_{2}\right)$ is the sub-matrix of $\mathbf{J}\left(\mathbf{w}_{2}\right)$ without the first row and the first column. Therefore, assuming that $\mathbf{w}_{2}$ is LAS in $\mathcal{W}_{2}$ (which is equivalent to the statement that $\mathbf{J}_{1,1}\left(\mathbf{w}_{2}\right)$ has only negative real part eigenvalues), $\mathbf{w}_{2}$ is LAS in $\mathcal{W} \backslash \mathcal{X}_{2}$ as well if and only if $R_{1}\left(\mathbf{w}_{2}\right)<0$ that is equivalent to $\varrho_{1}<-m_{1,2} x_{2}$. Likewise and by permutation, $\mathbf{w}_{1}$ is LAS in $\mathcal{W} \backslash \mathcal{X}_{1}$ provided that is LAS in $\mathcal{W}_{1}$ if and only if $\varrho_{2}<-m_{2,1} x_{1}$.

It is straightforward that (1) can show bistability in $\mathcal{W}$. Indeed, in the simplest case of $n=0$, the matrices $\mathbf{J}\left(\mathbf{w}_{1}\right)$ and $\mathbf{J}\left(\mathbf{w}_{2}\right)$ become

$$
\left[\begin{array}{cc}
-\varrho_{1} & -\frac{\eta_{1,2}}{\eta_{1,1}} \varrho_{1} \\
0 & \varrho_{2}-\frac{\eta_{2,1}}{\eta_{1,1}} \varrho_{1}
\end{array}\right] \text { and }\left[\begin{array}{cc}
\varrho_{1}-\frac{\eta_{1,2}}{\eta_{2,2}} \varrho_{2} & 0 \\
-\frac{\eta_{2,1}}{\eta_{2,2}} \varrho_{2} & -\varrho_{2}
\end{array}\right]
$$

respectively. Therefore, $\mathbf{w}_{1}$ and $\mathbf{w}_{2}$ are both feasible and LAS whenever $\left(0<\varrho_{2}<\frac{\eta_{2,1}}{\eta_{1,1}} \varrho_{1}\right) \wedge\left(0<\varrho_{1}<\frac{\eta_{1,2}}{\eta_{2,2}} \varrho_{2}\right)$. (Note that for $n \geq 1$, bistability in $\mathcal{W}$ can easily be found numerically.)

Nonetheless, the necessary and sufficient condition for the non-trivial fixed points to be LAS cannot be determined in the most general case. For this reason, given an intial condition, the long term behaviour of the solution of system (1) cannot be predicted in the general case unless an identified global asymptotic stability condition is satisfied. 


\section{Global asymptotic stability condition}

If deriving the global asymptotic condition for the Lotka-Volterra model for two competing species on.

In the following, we focus on the case of the dimorphic fixed point $\mathbf{w}_{3}$ and show in the appendix that the global asymptotic stability condition that emerges also applies to the three other fixed points.

Inspired by the previous works $[4,30]$, we define $V$ as

$$
\begin{aligned}
V(\mathbf{w}):= & a_{1}\left(X_{1}-\left(1+\log \frac{X_{1}}{\xi_{1}}\right) \xi_{1}\right)+a_{2}\left(X_{2}-\left(1+\log \frac{X_{2}}{\xi_{2}}\right) \xi_{2}\right) \\
& +\frac{1}{2} \sum_{p=1}^{n} b_{p}\left(Y_{p}-\frac{1}{v_{p}}\left(u_{1, p} \xi_{1}+u_{2, p} \xi_{2}\right)\right)^{2},
\end{aligned}
$$

where $a_{1}, a_{2}, b$ are arbitrary positive real numbers.

The function $V$ is obviously continuously differentiable on $\mathcal{W}^{\star}$. Moreover, its first two terms are of the form $h(x):=x-c-c(\log x-\log c)$ with $c>0$. Given that the first derivative of $h$ is $h^{\prime}(x)=1-\frac{c}{x}$ and its second derivative is $f^{\prime \prime}(x)=\frac{c}{x^{2}}>0$, it follows that $\underset{\mathbb{R}_{+}^{\star}}{\operatorname{argmin}}(f)=c$ and $\min _{\mathbb{R}_{+}^{\star}}(f)=0$. Therefore, and since $V$ is a separable function in each component of $\mathbf{w}$, it follows that $V(\mathbf{w})>0$ for all $\mathbf{w} \in \mathcal{W}^{\star} \backslash\left\{\mathbf{w}_{3}\right\}$ and $V\left(\mathbf{w}_{3}\right)=0$, condition $(\alpha)$ holds.

Before calculating the time derivative of $V$, let us remind that $R_{i}\left(\mathbf{w}_{3}\right)=0$ for $i=1,2$. Therefore, the following holds for all $\mathbf{w} \in \mathcal{W}$, and $i=1,2$,

$$
\begin{aligned}
R_{i}(\mathbf{w}) & =R_{i}(\mathbf{w})-R_{i}\left(\mathbf{w}_{3}\right), \\
& =\eta_{i, 1}\left(X_{1}-\xi_{1}\right)+\eta_{i, 2}\left(X_{2}-\xi_{2}\right)+\sum_{p=1}^{n} \gamma_{i, p}\left(Y_{p}-\frac{1}{v_{p}}\left(u_{1, p} \xi_{1}+u_{2, p} \xi_{2}\right)\right) .
\end{aligned}
$$


It follows from here that

$$
\begin{aligned}
& \frac{\mathrm{d} V}{\mathrm{~d} t}(\mathbf{w})= a_{1}\left(X_{1}-\xi_{1}\right) R_{1}(\mathbf{w})+a_{2}\left(X_{2}-\xi_{2}\right) R_{2}(\mathbf{w}) \\
&+\sum_{p=1}^{n} b_{p}\left(Y_{p}-\frac{1}{v_{p}}\left(u_{1, p} \xi_{1}+u_{2, p} \xi_{2}\right)\right)\left(u_{1, p} X_{1}+u_{2, p} X_{2}-v_{p} Y_{p}\right) \\
&= \sum_{i=1}^{2} a_{i} \eta_{i, 1}\left(X_{i}-\xi_{i}\right)\left(X_{1}-\xi_{1}\right)+a_{i} \eta_{i, 2}\left(X_{i}-\xi_{i}\right)\left(X_{2}-\xi_{2}\right) \\
&+\sum_{p=1}^{n}\left(\left(a_{1} \gamma_{1, p}+b_{p} u_{1, p}\right)\left(Y_{p}-\frac{1}{v_{p}}\left(u_{1, p} \xi_{1}+u_{2, p} \xi_{2}\right)\right)\left(X_{1}-\xi_{1}\right)\right. \\
&+\left(a_{2} \gamma_{2, p}+b_{p} u_{2, p}\right)\left(Y_{p}-\frac{1}{v_{p}}\left(u_{1, p} \xi_{1}+u_{2, p} \xi_{2}\right)\right)\left(X_{2}-\xi_{2}\right) \\
&\left.\quad-b_{p} v_{p}\left(Y_{p}-\frac{1}{v_{p}}\left(u_{1, p} \xi_{1}+u_{2, p} \xi_{2}\right)\right)^{2}\right)
\end{aligned}
$$

which is equivalent to the following quadratic form

$$
\frac{\mathrm{d} V}{\mathrm{~d} t}(\mathbf{w})=\frac{1}{2}\left(\mathbf{w}-\mathbf{w}_{3}\right)^{\mathrm{T}} \cdot\left(\mathbf{D} \cdot \mathbf{P}+\mathbf{P}^{\mathrm{T}} \cdot \mathbf{D}\right) \cdot\left(\mathbf{w}-\mathbf{w}_{3}\right),
$$

where $\mathbf{D}$ is the diagonal matrix $\operatorname{diag}\left(a_{1}, a_{2}, b_{1}, \ldots, b_{p}\right)$ and

$$
\mathbf{P}=\left[\begin{array}{ccccc}
\eta_{1,1} & \eta_{1,2} & \gamma_{1,1} & \ldots & \gamma_{1, n} \\
\eta_{2,1} & \eta_{2,2} & \gamma_{2,1} & \ldots & \gamma_{2, n} \\
u_{1,1} & u_{2,1} & -v_{1} & \mathbf{0}_{p-2}^{\mathrm{T}} & 0 \\
\vdots & \vdots & \mathbf{0}_{p-2} & \ddots & \mathbf{0}_{p-2} \\
u_{1, n} & u_{2, n} & 0 & \mathbf{0}_{p-2}^{\mathrm{T}} & -v_{p}
\end{array}\right]
$$

It follows from (9) that property $(\beta)$ holds if and only if $\mathbf{D} . \mathbf{P}+\mathbf{P}^{\mathrm{T}} . \mathbf{D}$ is negative definite (which is equivalent to the negativity of its associated quadratic form).

A theorem from [31](p. 137) allows the existence of such a positive diagonal matrix D provided that the matrix $\mathbf{P}$ is strictly row diagonally dominant. It formally states that

$$
\begin{aligned}
& \forall q \in \mathbb{N}^{\star}, \forall \mathbf{A}=\left(a_{i, j}\right)_{(i, j) \in\{1, \ldots, q\}^{2}}, \\
& (\operatorname{diag}(\mathbf{A}) \succ 0) \wedge\left(\exists\left(k_{j}\right)_{j \in\{1, \ldots, q\}}>0: \forall i \in \llbracket 1 ; q \rrbracket, k_{i} a_{i, i}>\sum_{j \in\{1, \ldots, q\} \backslash\{i\}} k_{j}\left|a_{i, j}\right|\right) \\
& \Longrightarrow \exists \mathbf{C}=\operatorname{diag}\left(c_{j}\right)_{j \in\{1, \ldots, q\}}>0:\left(\mathbf{C} . \mathbf{A}+\mathbf{A}^{\mathrm{T}} \cdot \mathbf{C}\right) \text { is positive definite. }
\end{aligned}
$$

Applying this theorem to $-\mathbf{P}$ with the particular set of positive real numbers $\left(k_{j}\right)_{j \in\{1, \ldots, p+2\}}:=$ 
$(\kappa, 1, \kappa, \ldots, \kappa)$ (the biological relevance of which is exposed in the discussion), it results that if there exists a scaling quantity $\kappa>0$ such that

$$
\begin{cases}-\eta_{1,1} & >\frac{1}{\kappa}\left|\eta_{1,2}\right|+\sum_{p=1}^{n}\left|\gamma_{1, p}\right|, \\ -\eta_{2,2} & >\kappa\left|\eta_{2,1}\right|+\kappa \sum_{p=1}^{n}\left|\gamma_{2, p}\right|, \\ v_{p} & >u_{1, p}+\frac{1}{\kappa} u_{2, p}, \quad \forall p \in\{1, \ldots, n\},\end{cases}
$$

then there exists $\left(a_{1}, a_{2}, b_{1}, \ldots, b_{p}\right) \in\left(\mathbb{R}_{+}^{\star}\right)^{p+2}$ that ensures property $(\beta)$.

Overall, provided that $\mathbf{w}_{3} \in \mathcal{W}^{\star}$, the set of inequalities (11) represents a sufficient condition for $V$ to be a Lyapunov function hence a sufficient condition for $\mathbf{w}_{3}$ to be GAS on $\mathcal{W}^{\star}$. In the appendix, we show that this condition also guarantees the global asymptotic stability of the three other fixed points in relevant subspaces of $\mathcal{W}$ provided that they are LAS in these spaces. 


\section{Discussion}

In this work we present an extension of the two-species Lotka-Volterra equations that captures indirect interactions through a potentially large number of diffusible and renewable compounds which are model [13]. Here, microbial type 1 is responsible for the high intra-type and the low competition geometric means, thus leaving a certain freedom to the traits of microbial type 2 . 


\section{Acknowledgements}

The author would like to thank Samuel Alizon, Yannis Michalakis, Yves Dumont and Alain Rapaport

200 for their helpful comments and the French Ministry of Higher Education and Research, CNRS and IRD for their support. 


\section{References}

[1] Grobman DM. Homeomorphism of systems of differential equations. Doklady Akademii Nauk SSSR. 1959;128(5):880-881.

[13] Barabás G, Michalska-Smith MJ, Allesina S. The Effect of Intra- and Interspecific Competition on Coexistence in Multispecies Communities. The American Naturalist. 2016;188(1):E1-E12. PMID: 27322128.

[3] Lyapunov AM. The general problem of the stability of motion. International Journal of Control. 1992;55(3):531-534.

[4] Goh BS. Global stability in many-species systems. The American Naturalist. 1977;p. 135-143.

[5] Hofbauer J, Sigmund K. Adaptive dynamics and evolutionary stability. Applied Mathematics Letters. 1990;3(4):75-79.

[6] Waage P, Guldberg CM. Studies concerning affinity. J Chem Educ. 1986;63(12):1044.

[7] McCallum H, Barlow N, Hone J. How should pathogen transmission be modelled? Trends in Ecology \& Evolution. 2001;16(6):295-300.

[8] Geritz SAH, Kisdi E, Meszena G, Metz JAJ. Evolutionarily singular strategies and the adaptative growth and branching of the evolutionary tree. Evolutionary Ecology. 1998;12:35-57.

[9] Metz JAJ, Staňková K, Johansson J. The canonical equation of adaptive dynamics for life histories: from fitness-returns to selection gradients and Pontryagin's maximum principle. Journal of Mathematical Biology. 2016;72(4):1125-1152.

[10] Lotka AJ. Elements of physical biology. Wiliams and Wilkins; 1925.

[11] Volterra V. Variations and fluctuations of the number of individuals in animal species living together. Journal du Conseil Permanent Internation pour l'Exploration de la Mer. 1928;3(1):351.

[12] Hernández-Bermejo B, Fairén V. Lotka-Volterra representation of general nonlinear systems. Mathematical Biosciences. 1997;140(1):1-32. 
[14] Pepper JW, Rosenfeld S. The emerging medical ecology of the human gut microbiome. Trends in Ecology \& Evolution. 2012;27(7):381-384.

[15] Coyte KZ, Schluter J, Foster KR. The ecology of the microbiome: Networks, competition, and stability. Science. 2015;350(6261):663-666.

[16] Begon M, Townsend CR, Harper JL. From individuals to ecosystems. Blackwell Publishers Hoboken; 2005.

[17] Hellard E, Fouchet D, Vavre F, Pontier D. Parasite-Parasite Interactions in the Wild: How To Detect Them? Trends in Parasitology. 2015;31(12):640-652.

[18] Bashey F. Within-host competitive interactions as a mechanism for the maintenance of parasite diversity. Philosophical Transactions of the Royal Society B: Biological Sciences. 2015;370(1675).

[19] Mideo N. Parasite adaptations to within-host competition. Trends in Parasitology. 2009;25(6):261-268.

[20] Neilands J. Siderophores: structure and function of microbial iron transport compounds. Journal of Biological Chemistry. 1995;270(45):26723-26726.

[21] Wandersman C, Delepelaire P. Bacterial iron sources: from siderophores to hemophores. Annual Review of Microbiology. 2004;58:611-647.

[22] Ghoul M, West SA, McCorkell FA, Lee ZB, Bruce JB, Griffin AS. Pyoverdin cheats fail to invade bacterial populations in stationary phase. Journal of Evolutionary Biology. 2016;

[23] Riley MA, Chavan MA. Bacteriocins. Springer-Verlag Berlin Heidelberg; 2007.

[24] Skaar EP. The battle for iron between bacterial pathogens and their vertebrate hosts. PLOS Pathogens. 2010;6(8):e1000949.

[25] Rath CM, Dorrestein PC. The bacterial chemical repertoire mediates metabolic exchange within gut microbiomes. Current Opinion in Microbiology. 2012;15(2):147-154.

[26] Dobson A, Cotter PD, Ross RP, Hill C. Bacteriocin production: a probiotic trait? Applied and Environmental Microbiology. 2012;78(1):1-6.

[27] Alizon S, Lion S. Within-host parasite cooperation and the evolution of virulence. Proceedings of the Royal Society B: Biological Sciences. 2011;278(1725):3738-3747. 
[28] Simonyi E, Kaszás M. Method for the dynamic analysis of nonlinear systems. Chemical Engineering. 1968;12(4):314-324.

[29] Zeeman ML. Hopf bifurcations in competitive three-dimensional Lotka-Volterra systems. Dynamics and Stability of Systems. 1993;8(3):189-216.

260 [30] Takeuchi Y. Global dynamical properties of Lotka-Volterra systems. World Scientific; 1996.

[31] Berman A, Plemmons RJ. Nonnegative matrices. The Mathematical Sciences, Classics in Applied Mathematics. 1979;9. 


\section{Appendix}

\section{A Global asymptotic stability of the null and monomorphic fixed points}

Here, we show that if conditions (11) are satisfied, then $\mathbf{w}_{0}, \mathbf{w}_{1}$ and $\mathbf{w}_{2}$ are GAS in their relevant spaces, namely $\mathcal{W} \cap \mathcal{X}_{2}, \mathcal{W} \cap \mathcal{X}_{1}$ and $\mathcal{W}$ for $\mathbf{w}_{0}$, and $\mathcal{W}_{i}$ and $\mathcal{W} \backslash \mathcal{X}_{i}$ for $\mathbf{w}_{i}, i=1,2$, as long as they belong and are LAS in these spaces. A distinct Lyapunov function has to be defined for each fixed point and the global asymptotic stability has to be separately investigated on each space.

Let us start by the null fixed point, for which one can consider the following function defined on $\mathbb{R}^{p+2}$

$$
V_{0}(\mathbf{w}):=a_{1} X_{1}+a_{2} X_{2}+\frac{1}{2} \sum_{p=1}^{n} b_{p} Y_{p}^{2}
$$

Property $\alpha$ clearly holds on $\mathcal{W}_{1}, \mathcal{W}_{2}$ and $\mathcal{W}$. As for property $\beta$, the calculation of $\frac{\mathrm{d} V_{0}}{\mathrm{~d} t}$ gives

$$
\begin{aligned}
& \frac{\mathrm{d} V_{0}}{\mathrm{~d} t}(\mathbf{w})= a_{1} R_{1}(\mathbf{w}) X_{1}+a_{2} R_{2}(\mathbf{w}) X_{2} \\
&+\sum_{p=1}^{n} b_{p} Y_{p}\left(u_{1, p} X_{1}+u_{2, p} X_{2}-v_{p} Y_{p}\right), \\
&=\sum_{i=1}^{2} a_{i} \varrho_{i} X_{i}+a_{i} \eta_{i, i} X_{i}^{2}+a_{i} \eta_{i, k} X_{i} X_{k} \\
&+\sum_{p=1}^{n}\left(\left(a_{1} \gamma_{1, p}+b_{p} u_{1, p}\right) Y_{p} X_{1}\right. \\
&+\left(a_{2} \gamma_{2, p}+b_{p} u_{2, p}\right) Y_{p} X_{2} \\
&\left.-b_{p} v_{p} Y_{p}^{2}\right),
\end{aligned}
$$

hence $\frac{\mathrm{d} V_{0}}{\mathrm{~d} t}(\mathbf{w})$ can be written as

$$
\frac{\mathrm{d} V_{0}}{\mathrm{~d} t}(\mathbf{w})=\frac{1}{2} \mathbf{w}^{\mathrm{T}} \cdot\left(\mathbf{D} \cdot \mathbf{P}+\mathbf{P}^{\mathrm{T}} \cdot \mathbf{D}\right) \cdot \mathbf{w}+a_{1} \varrho_{1} X_{1}+a_{2} \varrho_{2} X_{2} \cdot
$$

Now, let us recall from section 4 that $\mathbf{w}_{0}$ is LAS on $\mathcal{W}$ iff $\left(\varrho_{1}<0\right) \wedge\left(\varrho_{2}<0\right)$. Therefore, if $\mathbf{w}_{0}$ is LAS on $\mathcal{W}, \frac{\mathrm{d} V_{0}}{\mathrm{~d} t}(\mathbf{w})<\frac{1}{2} \mathbf{w}^{\mathrm{T}} \cdot\left(\mathbf{D} \cdot \mathbf{P}+\mathbf{P}^{\mathrm{T}} \cdot \mathbf{D}\right) \cdot \mathbf{w}$, hence if $(11)$ then $V_{0}$ is a Lyapunov function than proves the global asymptotic stability of $\mathbf{w}_{0}$ on $\mathcal{W}$.

If stability is investigated on $\mathcal{W} \cap \mathcal{X}_{k}$, then all occurrences of $X_{k}$ vanish, only $\varrho_{i}<0$ is required for local asymptotic stability and the quadratic form reduces to dimension $p+1$. The application of 
theorem (10) then provides sufficient conditions of the form

$$
\exists \kappa^{\prime}>0:\left(-\eta_{i, i}>\kappa^{\prime} \sum_{p=1}^{n}\left|\gamma_{i, p}\right|\right) \wedge\left(\kappa^{\prime} v_{p}>u_{i, p}, p=1, \ldots, n\right)
$$

with $\kappa^{\prime}>0$. It is straightforward to see that these conditions are weaker than, and therefore implied by, (11).

As for the monomorphic fixed points $\mathbf{w}_{i}, i=1,2$, one can consider the following function defined on $\mathcal{W}_{i} \backslash \mathcal{X}_{i}$

$$
V_{i}(\mathbf{w}):=a_{i}\left(X_{i}-\left(1+\log \frac{X_{i}}{x_{i}}\right) x_{i}\right)+a_{k} X_{k}+\frac{1}{2} \sum_{p=1}^{n} b_{p}\left(Y_{p}-\frac{u_{i, p}}{v_{p}} x_{i}\right)^{2} .
$$

It is straightforward to see that property $\alpha$ holds on $\mathcal{W}_{i}$ and $\mathcal{W} \backslash \mathcal{X}_{i}$.

The derivation of $V_{i}$ with respect to time leads to

$$
\begin{aligned}
\frac{\mathrm{d} V_{i}}{\mathrm{~d} t}(\mathbf{w})=a_{i} & \left(X_{i}-x_{i}\right) R_{i}(\mathbf{w})+a_{k} R_{k}(\mathbf{w}) X_{k} \\
& +\sum_{p=1}^{n} b_{p}\left(Y_{p}-\frac{u_{i, p}}{v_{p}} x_{i}\right)\left(u_{1, p} X_{1}+u_{2, p} X_{2}-v_{p} Y_{p}\right) .
\end{aligned}
$$

Restricting the investigation to $\mathcal{W}_{i}$, and applying relation (8) to $R_{i}(\mathbf{w})$, it follows that

$$
\begin{aligned}
\frac{\mathrm{d} V_{i}}{\mathrm{~d} t}(\mathbf{w})= & a_{i} \eta_{i, i}\left(X_{i}-x_{i}\right)^{2}+ \\
& \quad+\sum_{p=1}^{n}\left(\left(a_{i} \gamma_{i, p}+b_{p} u_{i, p}\right)\left(Y_{p}-\frac{u_{i, p}}{v_{p}} x_{i}\right)\left(X_{i}-x_{i}\right)-b_{p} v_{p}\left(Y_{p}-\frac{u_{i, p}}{v_{p}} x_{i}\right)^{2}\right),
\end{aligned}
$$

which is analogous to the aforementioned reduced quadratic form of dimension $p+1$. Hence, $V_{i}$ satisfies property $\beta$ on $\mathcal{W}_{i}$ under the conditions (13) which are implied by (11), that is $\mathbf{w}_{i}$ is GAS in $\mathcal{W}_{i}$, provided $\mathbf{w}_{i} \in \mathcal{W}_{i}$

Finally, coming back to $\mathcal{W} \backslash \mathcal{X}_{i}$ leads to $\frac{\mathrm{d} V_{i}}{\mathrm{~d} t}(\mathbf{w})=\frac{1}{2}\left(\mathbf{w}-\mathbf{w}_{i}\right)^{\mathrm{T}} \cdot\left(\mathbf{D} \cdot \mathbf{P}+\mathbf{P}^{\mathrm{T}} \cdot \mathbf{D}\right) \cdot\left(\mathbf{w}-\mathbf{w}_{i}\right)+$ $a_{k} R_{k}\left(\mathbf{w}_{i}\right) X_{k}$. Now recalling that $\mathbf{w}_{i}$ is LAS in $\mathcal{W} \backslash \mathcal{X}_{i}$ if and only if $\mathbf{w}_{i}$ is LAS in $\mathcal{W}_{i}$ and $R_{k}\left(\mathbf{w}_{i}\right)<0$, it results that if conditions (11) hold and $R_{k}\left(\mathbf{w}_{i}\right)=\varrho_{k}-\frac{m_{k, i}}{m_{i, i}} \varrho_{i}<0$, then $\frac{\mathrm{d} V_{i}}{\mathrm{~d} t}(\mathbf{w})<\frac{1}{2}\left(\mathbf{w}-\mathbf{w}_{i}\right)^{\mathrm{T}} \cdot\left(\mathbf{D} \cdot \mathbf{P}+\mathbf{P}^{\mathrm{T}} \cdot \mathbf{D}\right) \cdot\left(\mathbf{w}-\mathbf{w}_{i}\right)<0$, hence $\mathbf{w}_{i}$ is GAS in $\mathcal{W} \backslash \mathcal{X}_{i}$ as well. 